\title{
A STABLE CONVERSE TO THE VIETORIS-SMALE THEOREM WITH APPLICATIONS TO SHAPE THEORY ${ }^{1}$
}

\author{
BY
}

STEVE FERRY

\begin{abstract}
Our main result says that if $f: X \rightarrow Y$ is a map between finite polyhedra which has $k$-connected homotopy fiber, then there is an $n$ such that $f \times$ id: $X \times I^{n} \rightarrow Y$ is homotopic to a map with $k$-connected point-inverses. This result is applied to give an algebraic characterization of compacta shape equivalent to locally $n$-connected compacta. We also show that a $U V^{1}$ compactum can be "improved" within its shape class until its homotopy theory and strong shape theory are the same with respect to finite dimensional polyhedra.
\end{abstract}

1. Introduction. In this paper we study the problem of when a map $f: X \rightarrow Y$ between nice topological spaces is homotopic to a map with some form of the homotopy lifting property. If $f$ is a map between finite polyhedra, a necessary condition for $f$ to be homotopic to a Hurewicz fibration is that the homotopy fiber of $f$ be homotopy equivalent to a finite polyhedron. Thus, in order to achieve general results, we are forced to study much weaker lifting properties. We first prove that if the homotopy fiber of $f$ is $k$-connected, then $f$ is (stably) homotopic to a PL map with $k$-connected point-inverses. We use this result to prove that if the homotopy fiber of $f$ has finite skeleta (i.e. is homotopy equivalent to a $\mathrm{CW}$ complex with finite $n$-skeleton for each $n$ ) then $f$ is (stably) homotopic to a map with the approximate lifting property for $m$-dimensional spares. Here $m$ is fixed but as large as we desire.

In the sections on shape theory, we apply the results of the preceding sections to show that for many compact spaces $X$ it is possible to 'improve' $X$ within its shape class to obtain a compactum $X^{\prime}$ whose homotopy theory and (strong) shape theory are closely related.

We will now introduce some terminology and give more precise statements of our results. If $X$ is a compactum imbedded in an ANR $M, X$ is said to have property $U V^{k}, 0 \leqslant k<\infty$, if for each neighborhood $U$ of $X$ there is a neighborhood $V \subset U$ of $X$ such that the inclusion-induced map $\pi_{l}(V) \rightarrow \pi_{l}(U)$ is zero for $0 \leqslant l \leqslant k$. For $k=\infty$ we require that $V$ contract to a point in $U$. It is known (see, for example, [L]) that this property is a shape property of $X$ and is independent of

Received by the editors March 6, 1979.

AMS (MOS) subject classifications (1970). Primary 55F65, 57C05, 57C10; Secondary 55B05, 54F20, 54F40.

Key words and phrases. Vietoris-Smale theorem, $U V^{k}$-map, strong shape theory, Hilbert cube manifold, finiteness obstruction.

${ }^{1}$ Research partially supported by NSF grants and the A.P. Sloan Foundation. 
the particular imbedding. For $\mathrm{CW}$ complexes, property $U V^{k}$ reduces to the usual notion of $k$-connectedness. A map $f: X \rightarrow Y$ between compact ANR's is called a $U V^{k}$-map if for each $y \in Y, f^{-1}(y)$ is nonempty and has property $U V^{k}$. A $U V^{\infty}$ compactum is called cell-like (CE) and a $U V^{\infty}$-map is called a CE map. We now state a version of the Vietoris-Smale theorem of the title.

THEOREM [Sm]. If $X$ and $Y$ are compact $A N R$ 's and $f: X \rightarrow Y$ is a $U V^{k}$-map then $f_{*}: \pi_{l}(X) \rightarrow \pi_{l}(Y)$ is an isomorphism for $l \leqslant k$ and an epimorphism for $l=k+1$ (i.e., $f$ is $(k+1)$-connected).

Recall that the Hilbert cube $Q$ is the countable product of copies of the unit interval. A Hilbert cube manifold ( $Q$-manifold) is a separable metric space in which each point has an open neighborhood homeomorphic to an open subset of $Q$. Here is our stable converse to Smale's theorem.

THeOREM 1. If $M$ and $N$ are compact $Q$-manifolds and $f: M \rightarrow N$ is $(k+1)$ connected, $0 \leqslant k<\infty$, then $f$ is homotopic to a $U V^{k}$-map.

We remark that this theorem is false for $k=\infty$. If $f: M \rightarrow N$ is a nonsimple homotopy equivalence, then $f$ is not homotopic to a $\mathrm{CE}$ map [Ch]. We will now state a PL theorem which implies Theorem 1.

THEOREM $1^{\prime}$. If $K$ and $L$ are finite polyhedra and $f: K \rightarrow L$ is $a(k+1)$-connected map, $0 \leqslant k<\infty$, then there exist a polyhedron $K^{\prime}, a C E-P L$ map $c: K^{\prime} \rightarrow K$, and a $U V^{k}-P L$ map $f^{\prime}: K^{\prime} \rightarrow L$ such that $f \circ c \simeq f^{\prime}$.

In view of Chapman's proof that $\mathrm{CE}$ maps between polyhedra are simple homotopy equivalences it is natural, though perhaps naive, to ask whether a nonsimple homotopy equivalence can have highly connected point-inverses. Theorem $1^{\prime}$ shows that this is indeed possible.

If $X$ and $Y$ are compact metric ANR's, a map $p: X \rightarrow Y$ is said to have the approximate homotopy lifting property (AHLP) with respect to a compact space $Z$ if for every homotopy $f: Z \times I \rightarrow Y$, map $F_{0}: Z \rightarrow X$, such that $p \circ F_{0}=f \mid Z \times 0$, and $\varepsilon>0$ there is a map $F: Z \times I \rightarrow X$ such that $F_{0}=F \mid Z \times\{0\}$ and $d(p \circ F(z, t), f(z, t))<\varepsilon$ for each $(z, t) \in Z \times I . f$ is called an approximate fibration if $f$ has the AHLP for all compacta. We will call $f$ an $A F^{n}$-map if $f$ has the AHLP for $n$-dimensional compacta. Compare with [M-R]. If $f: X \rightarrow Y$ is a map, $X$, $Y$ ANR's and $Y$ connected, then the homotopy fiber of $f$ is the fiber of the mapping path fibration [S, p. 99] of $f$.

THEOREM 2. If $M$ and $N$ are compact $Q$-manifolds and the homotopy fiber of $f$ : $M \rightarrow N$ has finite skeleta, then for any $n f$ is homotopic to an $A F^{n}$-map.

This theorem also has a stronger PL version:

THEOREM 2'. If $K$ and $L$ are polyhedra and the homotopy fiber of $f$ has finite skeleta, then for each $n$ there exist a polyhedron $K^{\prime}, a C E-P L$ map $c: K^{\prime} \rightarrow K$, and a $P L A F^{n}$-map $f^{\prime}: K^{\prime} \rightarrow L$ such that $f \circ c \simeq f^{\prime}$. 
As an application of Theorem 2, we show that a large class of compacta can be 'improved' within their shape classes.

THEOREM 3. If $X$ is a $U V^{1}$ compactum then $X$ is shape equivalent to a compactum $X^{\prime}$ such that for every finite-dimensional compactum $Z$ :

(i) Every strong shape morphism $f: Z \rightarrow X^{\prime}$ contains a map.

(ii) If $f, g: Z \rightarrow X^{\prime}$ are maps which are equivalent as strong shape morphisms, then $f$ and $g$ are homotopic as maps.

A Theorem 3', whose statement is similar to that of Theorem 3, holds for compacta which are inverse limits of ANR's with progressively more highly connected bonding maps. We should mention that our construction unavoidably yields infinite-dimensional spaces $X^{\prime}$, so we have not imbedded the strong shape category in the homotopy category. We do not know if this is possible.

We show in $\$ 5$ that our improved compacta are nearly as nice locally as algebraic considerations allow. In particular, we show that the strange compacta of Edwards and Geoghegan are shape equivalent to compacta which are $L C^{k}$ for all $k$. We also prove:

THEOREM 4. A continuum $X$ is shape equivalent to an $L C^{n}$ continuum if and only if pro- $\pi_{l}(X)$ is stable for $0 \leqslant l \leqslant n$ and Mittag-Leffler for $l=n+1$.

The case $n=0$ of this theorem is due to Krasinkiewicz [K].

We would like to thank John Walsh for suggesting that we attempt to prove the converse Vietoris-Smale theorem for $Q$-manifolds. The analogous finite-dimensional problem has been attacked by Walsh and Wilson, [Wa $\mathbf{W},\left[\mathbf{W} \mathbf{a}_{\mathbf{2}}\right],\left[\mathbf{W i}_{\mathbf{1}}\right],\left[\mathbf{W i}_{\mathbf{2}}\right]$. The full converse is not true for maps between spheres. We would like to thank Gerard Venema for helpful conversations and we would particularly like to thank Ross Geoghegan for sharing his knowledge of shape theory.

2. The proofs of Theorems 1 and $1^{\prime}$. Before proving Theorem 1', we will show that this theorem implies Theorem 1. Let $f: M \rightarrow N$ be a $(k+1)$-connected map between $Q$-manifolds. By the triangulation theorem for $Q$-manifolds, there are polyhedra $K$ and $L$ such that $K \times Q \cong M$ and $L \times Q \cong N$. The map

$$
K \stackrel{\stackrel{\times 0}{\rightarrow} K \times Q \stackrel{f}{\rightarrow} L \times Q \stackrel{\text { proj }}{\rightarrow} L}{f} L
$$

is $(k+1)$-connected, so Theorem $1^{\prime}$ produces a finite polyhedron $K^{\prime}$, a CE map $c$ : $K^{\prime} \rightarrow K$, and a $U V^{k}$-map $f^{\prime}: K^{\prime} \rightarrow L$ such that $f \circ c \simeq f^{\prime}$. Crossing with $Q$, we can approximate $c \times$ id by a homeomorphism, so we obtain a homotopy commuting diagram where $\left(f^{\prime} \times \mathrm{id}\right) \circ h^{-1}$ is the required $U V^{k}$-map from $M$ to $N$.

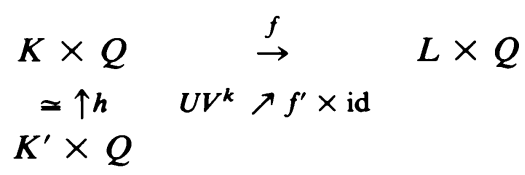

We now proceed with the proof of Theorem $1^{\prime}$. We will isolate an important special case of Theorem $1^{\prime}$ as Lemma 2.1. 
LEMMA 2.1. Let $K$ be a finite polyhedron and let $i: K \rightarrow K \cup_{f} D^{r+2}$ be the inclusion, where $D^{r+2}$ is a PL $(r+2)$-cell and $f: \partial D^{r+2} \rightarrow K$ is a PL map. There exist a finite polyhedron $K^{\prime}$, a CE-PL map $c: K^{\prime} \rightarrow K$, and a $U V^{r}$-map $i^{\prime}: K^{\prime} \rightarrow K$ such that $i \circ c \simeq i^{\prime}$.

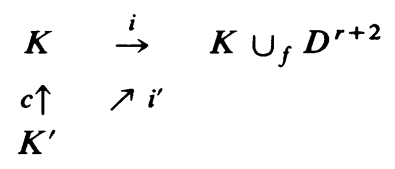

Proof. Let $K^{\prime}=M(f)$, the mapping cylinder of $f . c: K^{\prime} \rightarrow K$ is the mapping cylinder collapse, and $i^{\prime}: K^{\prime} \rightarrow K \cup_{f} D^{r+2}$ is a PL map which collapses the top of the mapping cylinder to a point. $i^{\prime}$ is $U V^{r}$ because the only noncontractible point-inverse of $i^{\prime}$ is a copy of $S^{r+1}$.

In proving Theorem $1^{\prime}$ we will work with finite $\mathrm{CW}$ complexes built up using PL cells and PL attaching maps. Thus, all of our spaces will be polyhedra. By construction, our maps will be PL. We will use the following well-known lemma frequently.

LEMMA 2.2. If $f: K_{1} \rightarrow L$ and $g: K_{2} \rightarrow L$ are $P L$ maps, then the pullback $P$ of $f$ and $g$ is a polyhedron. The induced maps $g^{\prime}: P \rightarrow K_{1}$ and $f^{\prime}: P \rightarrow K_{2}$ are $P L$. If $f$ is $U V^{k}$, then so is $f^{\prime}$.

Proof. Let $G_{1}=\left\{(x, y, z) \in K_{1} \times K_{2} \times L \mid f(x)=z\right\}$ and let $G_{2}=\{(x, y, z) \in$ $\left.K_{1} \times K_{2} \times L \mid g(y)=z\right\} . G_{1}$ and $G_{2}$ are polyhedra, since the graphs of $f$ and $g$ are polyhedra. $G_{1} \cap G_{2}=\{(x, y, z) \mid f(x)=g(y)=z\}$ is therefore a polyhedron and is homeomorphic to the desired pullback. The induced maps are restrictions of PL projection maps and are therefore PL. Each point-inverse of $f^{\prime}$ is homeomorphic to some point-inverse of $f$, so the last statement follows.

Proof (Theorem $1^{\prime}$ ). If $f: K \rightarrow L$ is $(k+1)$-connected, then $\pi_{l}(M(f), K)=0$ for $0 \leqslant l \leqslant k+1$, where $M(f)$ is the simplicial mapping cylinder of $f$. By Whitehead's cell-trading lemma ([Wh, p. 246] or [Co, 7.3]) there exist a polyhedron $Z$ and CE-PL maps $c_{1}: Z \rightarrow M(f), c_{2}: Z \rightarrow M$ where $M$ is a finite PL cell complex obtained from $K$ by attaching PL cells of dimension $\geqslant k+2$.

We write $K=K_{0} \subset K_{1} \subset \ldots \subset K_{n}=M$ where each $K_{i+1}$ is obtained from $K_{i}$ by attaching a PL $r$-cell, $r \geqslant k+2$. We will first use induction on $n$ to construct a finite polyhedron $K^{\prime \prime}$, a CE-PL map $c^{\prime \prime}: K^{\prime \prime} \rightarrow K$, and a PL $U V^{k}$-map $i^{\prime \prime}: K^{\prime \prime} \rightarrow M$ such that $i \circ c^{\prime \prime} \simeq i^{\prime \prime}$, where $i: K \rightarrow M$ is the inclusion.

The case $n=0$ is trivial. We assume that there exist CE-PL maps $c^{\prime \prime \prime}: K^{\prime \prime \prime} \rightarrow K$ and $U V^{k}$-PL $i^{\prime \prime \prime}: K^{\prime \prime \prime} \rightarrow K_{n-1}$ such that the composition of $c^{\prime \prime \prime}$ with the inclusion $i_{n-1}: K \rightarrow K_{n-1}$ is homotopic to $i^{\prime \prime \prime}$. By Lemma 2.1 , there exist a polyhedron $K^{\prime \prime \prime \prime}$, a CE-PL map $c^{\prime \prime \prime \prime}: K^{\prime \prime \prime \prime} \rightarrow K_{n-1}$, and a $U V^{k}$-PL map $i^{\prime \prime \prime \prime}: K^{\prime \prime \prime \prime} \rightarrow M$ such that the appropriate diagram homotopy commutes. The desired polyhedron $K^{\prime \prime}$ is the pullback of $i^{\prime \prime \prime}$ and $c^{\prime \prime \prime \prime} . c^{\prime \prime}$ is the composition of $c^{\prime \prime \prime}$ with the induced CE-PL map $K^{\prime \prime} \rightarrow K^{\prime \prime \prime}$ and $i^{\prime \prime}$ is the composition of the induced $U V^{k}$-PL map $K^{\prime \prime} \rightarrow K^{\prime \prime \prime \prime}$ with $i^{\prime \prime \prime \prime}$. Note that the Vietoris-Smale theorem implies that the composition of $U V^{k}-\mathrm{PL}$ maps is $U V^{k}-\mathrm{PL}$. 
We now have a homotopy commuting diagram.

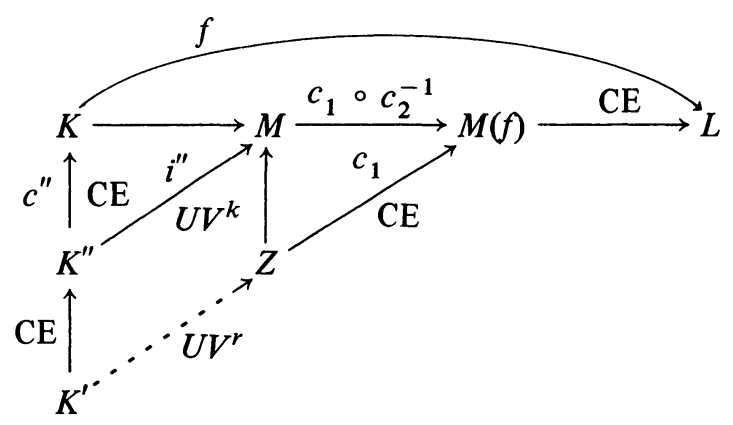

We complete the proof of Theorem 1 by letting $K^{\prime}$ be the pullback of $i^{\prime \prime}$ and $c_{2}$. $c^{\prime}$ is the composition of $c^{\prime \prime}$ with the induced CE map $K^{\prime} \rightarrow K^{\prime \prime}$ and $i^{\prime}$ is the composition of the induced $U V^{k}$-map with $c_{1}$ and the mapping cylinder collapse.

3. The proof of Theorem $2^{\prime}$. The proof of Theorem $2^{\prime}$ will rely on the following characterization of $A F^{n}$-maps which is a specialized version of a theorem [D-T, Satz 2.7] of Dold and Thom. See [C-D] for a Čech version.

THEOREM. Let $f: K \rightarrow L$ be a PL map between finite polyhedra. $f$ is an $A F^{n}$-map if the following condition holds: For each $x \in L$ there are arbitrarily small contractible neighborhoods $U$ of $x$ such that for each $y \in U$ the inclusion $f^{-1}(y) \rightarrow f^{-1}(U)$ induces isomorphisms on homotopy groups through dimension $n$.

Our strategy in proving Theorem $2^{\prime}$ will be to construct a homotopy commuting diagram below with $p^{\prime \prime}$ a PL $A F^{N}$-map and $s N$-connected for $N$ large. We will then apply Theorem $1^{\prime}$ to the map $s$. We begin with the following lemma.

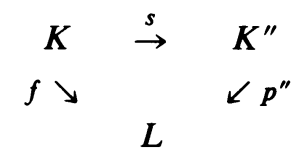

LeMma 3.1. Let $B$ be a connected finite $P L$ cell complex and let $\mathscr{P}: \mathcal{E} \rightarrow B$ be $a$ Hurewicz fibration from an $A N R$ to $B$ with fiber $\mathscr{F}$. If $\mathcal{F}$ has finite skeleta then for each $n$ there exist a finite polyhedron $K, a P L A F^{n}$-map $p: K \rightarrow B$, and an $n$-connected map $h: K \rightarrow \mathcal{E}$ such that $\mathscr{P} \circ h=p$.

Proof. The lemma is trivial if $B$ is a point. We will proceed by induction on PL cells. Assume that $B=B^{\prime} \cup D^{r}$, that $B^{\prime}$ is connected (since $B$ is connected we can assume that there is only one zero cell-an easy pullback construction shows that if Lemma 3.1 is true for $B$ then it is true for any space homotopy equivalent to $B$ ), and that we have the commutative diagram below in which $p^{\prime}$ is an $A F^{n}$-map and the restriction of $h^{\prime}$ to any fiber is $n$-connected.

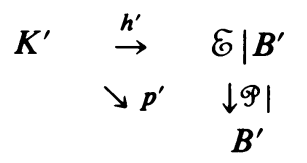


Write $D^{r}=S^{r-1} \times[0,1] \cup D_{1}^{r}$. By pulling $p^{\prime}$ back over the collapse $S^{r-1} \times[0,1]$ $\cup B^{\prime} \searrow B^{\prime}$, we reduce to the case in which $\partial D^{r}$ is imbedded in $B^{\prime}$. Let $F_{1}$ be a $C W$ complex with finite skeleta which is homotopy equivalent to $\mathscr{F}$. Since $\mathcal{E} \mid \partial D^{r}$ extends over $D^{r}$, there is a map $t: \mathcal{E} \mid \partial D^{r} \rightarrow F_{1}$ which restricts to a homotopy equivalence on each fiber. The map $t \circ h^{\prime} \mid\left(p^{\prime}\right)^{-1}\left(\partial D^{r}\right):\left(p^{\prime}\right)^{-1}\left(\partial D^{r}\right) \rightarrow F_{1}$ is homotopic to a cellular map and therefore is homotopic to a map whose image lies in the $N$-skeleton $F_{1}^{(N)}$ of $F_{1}$ for some large $N$. Let $F$ be a polyhedron homotopy equivalent to $F_{1}^{(N)}$. There is a PL map (coming from the trivialization $t$ ) $T$ : $\left(p^{\prime}\right)^{-1}\left(\partial D^{r}\right) \rightarrow F$ whose restriction to each point-inverse of $p^{\prime}$ is $n$-connected. The desired polyhedron $K$ is $K^{\prime} \cup M(T)$ which projects onto $B$ by the map which comes from considering $D^{n}$ to be the cone on $\partial D^{n}$. That $h^{\prime}$ extends to $h: K \rightarrow \mathcal{E}$ is clear from the construction, since the trivialization of $\mathcal{E} \mid D^{r}$ was used to construct $T$. That $p$ is an $A F^{n}$-map is clear from the criterion at the beginning of this section. That $h$ is highly connected follows from the fact that a PL $A F^{n}$-map satisfies the homotopy sequence of a fibration through degree $n$.

We will now proceed with the proof of Theorem $2^{\prime}$. Let $f: K \rightarrow L$ be a map between finite polyhedra as in the statement of Theorem $2^{\prime}$. Let $\mathscr{P}: \mathcal{E} \rightarrow L$ be the mapping path fibration of $f$. Let $N \gg \operatorname{dim} K$. According to Lemma 3.1, there exist a finite polyhedron $K^{\prime \prime}$, an $A F^{N}$-map $p^{\prime \prime}: K^{\prime \prime} \rightarrow L$, and a map $h^{\prime \prime}: K^{\prime \prime} \rightarrow \mathcal{E}$ such that $\mathscr{P} \circ h^{\prime \prime}=p^{\prime \prime}$ and such that $h^{\prime \prime}$ is $N$-connected. We therefore have a homotopy commuting diagram.

\begin{tabular}{|c|c|c|}
\hline & $d$ & \\
\hline$\underset{K}{\simeq}$ & $\varepsilon$ & $\stackrel{h^{\prime \prime}}{\leftarrow} K^{\prime \prime}$ \\
\hline$f \searrow$ & $\begin{array}{c}\mathcal{P} \downarrow \\
L\end{array}$ & $\swarrow p^{\prime \prime}$ \\
\hline
\end{tabular}

The map $d$ from $K^{\prime \prime}$ to $K$ is a homotopy domination since the obstructions to finding a homotopy section of $d$ lie in groups $H^{l}\left(K, \pi_{l-1}\left(\mathscr{F}_{1}\right)\right)$, where $\mathscr{F}_{1}$ is the homotopy fiber of $d$. $\mathscr{F}_{1}$ is $N-1$ connected, so these groups are all zero. If $s$ : $K \rightarrow K^{\prime \prime}$ is a map such that $d \circ s \simeq \mathrm{id}$, then $s$ is $(N-1)$-connected, so Theorem $1^{\prime}$ produces a polyhedron $K^{\prime}$, a CE-PL map $c: K^{\prime} \rightarrow K$, and a $U V^{N-2}$-PL map $s^{\prime}$ : $K^{\prime} \rightarrow K^{\prime \prime}$ such that $s \circ c \simeq s^{\prime}$. The desired polyhedron and CE map are $K^{\prime}$ and $c$. The desired map $f^{\prime}$ is $p^{\prime \prime} \circ s^{\prime}$, which is an $A F^{N-2}$-map.

LEMMA 3.2. ${ }^{2}$ If $K$ and $L$ are finite polyhedra with $\pi_{1} K=0=\pi_{1} L$ and $f: K \rightarrow L$ is a map, then the homotopy fiber of $\mathcal{F}$ has finite skeleta.

Proof. According to Wall [W, p. 61] it suffices to show that the group ring $\Lambda$ of $\pi_{1}(\mathscr{F})$ is Noetherian and that $H_{n}(\tilde{\mathscr{F}})$ is a finitely generated $\Lambda$-module for each $n$.

The homology groups of $K$ and $L$ are finitely generated. By the Hurewicz theorem modulo Serre classes of Abelian groups [S, p. 509], the homotopy groups of $K$ and $L$ are finitely generated Abelian groups in each dimension. It follows from the homotopy sequence of the mapping path fibration that $\pi_{1}(\mathscr{F})$ is a finitely generated Abelian group and that $\pi_{n}(\mathscr{F})=\pi_{n}(\tilde{\mathscr{F}})$ is a finitely generated Abelian

\footnotetext{
${ }^{2}$ This also holds for finite fundamental groups.
} 
group for each $n$. A second application of the mod- $\bigodot$ Hurewicz theorem shows that $H_{n}(\tilde{\mathscr{F}})$ is a finitely generated Abelian group for each $n$. According to Wall [W, p. 61] the group ring of a finitely generated Abelian group is Noetherian. This completes the proof.

Theorem 2 follows from Theorem $2^{\prime}$ as Theorem 1 follows from Theorem $1^{\prime}$.

Corollary $3.3 .^{3}$ If $M$ and $N$ are compact simply connected $Q$-manifolds and $f$ : $M \rightarrow N$ is a map then for each $n f$ is homotopic to an $A F^{n}$-map.

There is, of course, an analogous PL Corollary 3.3' whose statement we leave to the reader.

4. A lemma concerning inverse limits. In order to apply Theorems 1 and 2 to the study of compacta, we will need the following easy lemma.

LeMMA 4.1. Let $X_{1} \stackrel{f_{2}}{\leftarrow} X_{2} \stackrel{f_{3}}{\leftarrow} X_{3} \stackrel{f_{4}}{\leftarrow} \ldots$ be an inverse sequence of compact metric spaces and let $X=\lim \left(X_{i}, f_{i}\right)$. There is a sequence $\left\{\varepsilon_{i}\right\}_{i=1}^{\infty}$ of positive real numbers such that for any $N$ and any space $Z$ a sequence of maps $\alpha_{i}: Z \rightarrow X_{i}$ such that $d\left(f_{i} \circ \alpha_{i}, \alpha_{i-1}\right)<\varepsilon_{i-1}$ for all $i>N$ determines a map $\alpha: Z \rightarrow X$.

Proof. For each $n$, consider the sequence of maps

$$
\left\{f_{n+1} \circ \cdots \circ f_{n+k} \circ \alpha_{n+k}: Z \rightarrow X_{n}\right\}_{k=1}^{\infty} \text {. }
$$

The distance between the $k$ th and $(k+1)$ st maps in this sequence is

$$
d\left(f_{n+1} \circ \cdots \circ f_{n+k} \circ\left(f_{n+k+1} \circ \alpha_{n+k+1}\right), f_{n+1} \circ \cdots \circ f_{n+k} \circ\left(\alpha_{n+k}\right)\right) .
$$

If $\varepsilon_{n+k}$ is small enough, depending on the modulus of continuity of $f_{n+1} \circ \cdots \circ f_{n+k}$, this distance will be very small. Thus, for a suitable choice of $\left\{\varepsilon_{i}\right\}$, the sequence of maps described above will be Cauchy for each $n$ and each sequence will converge to a map $\alpha_{n}^{\prime}: Z \rightarrow X_{n}$. It is easy to verify that $f_{n} \circ \alpha_{n}^{\prime}=$ $\alpha_{n-1}^{\prime}$, so the sequence $\left\{\alpha_{i}^{\prime}\right\}$ induces a map $\alpha: Z \rightarrow X$ defined by $\alpha(z)=$ $\left(\alpha_{1}^{\prime}(z), \alpha_{2}^{\prime}(z), \ldots\right)$ for all $z$.

This lemma is a version 'for maps' of a well-known theorem of Morton Brown [B]. Our first proof of this result used Brown's theorem. The next corollary will be superceded by the results of $\$ 5$. It is included here because it exhibits the main idea of $\$ 5$ without getting the reader bogged down in the technicalities associated with strong shape theory.

COROllary. 4.2. If $X=\lim _{\leftarrow}\left(K_{i}, f_{i}\right), K_{i}$ a finite polyhedron, and

(i) each $f_{i}$ is an approximate fibration, then for every compact $Z$ each shape morphism $Z \rightarrow X$ is represented by a map;

(ii) each $f_{i}$ is an $A F^{i}$-map, then for each compact finite-dimensional $Z$ each shape morphism $Z \rightarrow X$ is represented by a map.

\footnotetext{
${ }^{3}$ Again, this holds for finite fundamental groups.
} 
Proof. A shape morphism $Z \rightarrow X$ is represented by maps $\alpha_{i}: Z \rightarrow K_{i}$ such that $f_{i} \circ \alpha_{i} \simeq \alpha_{i-1}$. Altering each $\alpha_{i}$ by a homotopy does not change the shape morphism. In either case, given a sequence $\left\{\varepsilon_{i}\right\}$ of positive numbers, we will construct maps $\left\{\alpha_{i}^{\prime}\right\}$ as in Lemma 4.1 .

Consider case (ii). Let $N=\operatorname{dim} Z$. Let $\alpha_{i}^{\prime}=\alpha_{i}$ for $i<N$. Using the $A F^{N}$ property of $f_{N}$, we can 'cover' the homotopy from $\alpha_{N-1}=\alpha_{N-1}^{\prime}$ to $f_{N} \circ \alpha_{N}$ by a homotopy from $\alpha_{N}$ to a new map $\alpha_{N}^{\prime}$ such that $f_{N} \circ \alpha_{N}^{\prime}$ is $\varepsilon_{N-1}$-close to $\alpha_{N-1}^{\prime}$. We construct $\alpha_{N+1}^{\prime}$ in a similar fashion, using the $A F^{N+1}$-property of $f_{N+1}$ and continue. By Lemma 4.1 or, better, by its proof, we are done.

The proof above will not generalize to show that maps into $X$ which are equivalent as shape maps are homotopic as maps. The interested reader is urged to work out the case of maps of a point into the dyadic solenoid as an exercise.

If $X$ is a $U V^{1}$ compactum, then $X$ can be written as an inverse limit $X$ $=\lim \left(K_{i}, f_{i}\right)$ with $\pi_{1}\left(K_{i}\right)=0$ for each $i$. By Corollary 3.3, $f_{i} \times$ id: $K_{i} \times Q \rightarrow K_{i-1}$ $\times \overleftarrow{Q}$ is homotopic to an $A F^{i}$-map $g_{i}$ for each $i . X^{\prime}=\lim \left(K_{i} \times Q, g_{i}\right)$ has the shape of $X$ and condition (ii) of Corollary 4.2 applies. Thus, every $U V^{1}$ compactum $X$ can be 'improved' within its shape class to a compactum $X^{\prime}$ for which shape maps $Z \rightarrow X^{\prime}, Z$ compact and finite dimensional, are represented by maps.

There is an interpretation of Lemma 4.1 which will be useful in the sequel. If $\left\{\left(X_{i}, f_{i}\right)\right\}_{i=1}^{\infty}$ is an inverse sequence of compacta, we can form a new inverse sequence $\left\{\left(X_{i}^{\prime}, f_{i}^{\prime}\right)\right\}_{i=1}^{\infty}$ of compacta by letting $X_{k}^{\prime}$ be the disjoint union $\amalg_{i=1}^{k} X_{i}$ and letting $f_{i}^{\prime}: X_{i}^{\prime} \rightarrow X_{i-1}^{\prime}$ be the map induced by identity maps and $f_{i}$. $\lim _{\leftarrow}\left(X_{i}^{\prime}, f_{i}^{\prime}\right)$ is a compactification of $\amalg_{i=1}^{\infty} X_{i}^{\prime}$ obtained by adding on a copy of $\lim _{\leftarrow}^{\leftarrow}\left(X_{i}, f_{i}\right)$. The condition that a sequence of maps $\alpha_{i}: Z \rightarrow X_{i}$ converge to a map $\alpha: \overleftarrow{Z} \rightarrow \underset{\leftarrow}{\lim }\left(X_{i}, f_{i}\right)$ is precisely the condition which appears in the proof of Lemma 4.1.

5. Approaching maps and strong shape. The reader who has worked out the suggested exercise involving the dyadic solenoid has discovered that it is not sufficient for our purposes to call sequences of maps $\left\{\alpha_{i}\right\}$ and $\left\{\beta_{i}\right\}, \alpha_{i}, \beta_{i}: Z \rightarrow X_{i}$ homotopic if there are independent level-preserving homotopies $h_{i}: \alpha_{i} \simeq \beta_{i}$. The idea of strong shape theory is to require some form of coherence between the various homotopies. Several equivalent notions of strong shape theory have been defined ([E-H], [D-S], [K-O], [Q]). We will use a slight modification of the construction of $[\mathbf{Q}]$, where strong shape theory is defined based on a modification of Borsuk's definition of fundamental sequence. Quigley's theory is further developed in [K-O].

DeFinition 5.1. If $X$ and $Y$ are compact metric spaces and $i: Y \rightarrow W$ is an imbedding, $W$ an absolute retract (AR), then an approaching map $f: X \rightarrow Y$ is a pair $(f, i)$ where $f$ is a map $f: X \times[0, \infty) \rightarrow W$ such that for each neighborhood $U$ of $i(Y)$ in $W$ there is an $N$ such that $f(X \times[N, \infty)) \subset U$. Two approaching maps $f$, $g: X \rightarrow Y(f=(f, i), g=(g, i))$ are homotopic if there is an approaching map $\underline{H}$ : $\bar{X} \times I \rightarrow Y \overline{(} \underline{H}=(H, \bar{i}))$ such that $\underline{H} \mid X \times\{0\}=f$ and $\underline{H} \mid X \times\{1\}=g$.

If $i^{\prime}: Y \rightarrow W^{\prime}$ is a second imbedding of $Y$ in an AR, then there are maps $t$ : $W \rightarrow W^{\prime}$ and $t^{\prime}: W^{\prime} \rightarrow W$ extending $i^{\prime} \circ i^{-1} \mid i(Y)$ and $i \circ\left(i^{\prime}\right)^{-1} \mid i^{\prime}(Y)$. Composing 
an approaching map $f: X \times[0, \infty) \rightarrow W$ with $t$ yields an approaching map $f^{\prime}:$ $t \circ f: X \times[0, \infty) \rightarrow W^{\prime}$. Since $W^{\prime}$ is an AR, the space of maps $t: W \rightarrow W^{\prime}$ extending $i^{\prime} \circ i^{-1} \mid i(Y)$ is contractible, so the association of $t \circ f$ to $f$ is independent of the choice of $t$ up to homotopy. If $[f]$ and $\left[f^{\prime}\right]$ are homotopy classes of approaching maps $X \rightarrow Y$ in AR's $W$ and $W^{\prime}$, we will say that $[f]$ and $\left[f^{\prime}\right]$ are equivalent if $[t f]=\left[f^{\prime}\right]$ for some $t$ as above. A strong shape morphism $\underline{s}: X \stackrel{-}{\rightarrow} Y$ is an equivalence class of homotopy classes of approaching maps from $X$ to $Y$. In practice, then, a strong shape morphism will be a homotopy class of approaching maps where $i: Y \rightarrow W$ is an imbedding of $Y$ into some convenient AR.

Definition 5.2. If $\left\{\left(X_{i}, f_{i}\right)\right\}$ is a finite or infinite inverse system, we will define $\operatorname{Map}\left\{\left(X_{i}, f_{i}\right)\right\}$ to be the union of the mapping cylinders $M\left(f_{i}\right)$ with the base of $M\left(f_{i+1}\right)$ identified with the top of $M\left(f_{i}\right)$. There is a natural map $p: \operatorname{Map}\left\{\left(X_{i}, f_{i}\right)\right\} \rightarrow$ $R^{1}$ which sends each $X_{i}$ to $i$ and interpolates linearly between the various $X_{i}$ 's. If $\left\{\left(X_{i}, f_{i}\right)\right\}_{i=1}^{\infty}$ is an infinite inverse system, we can define a new inverse system $\left\{\left(X_{i}^{\prime}, f_{i}^{\prime}\right)\right\}_{i=0}^{\infty}$ by setting $X_{k}^{\prime}=\operatorname{Map}\left(\mathrm{pt} \leftarrow X_{1} \leftarrow X_{2} \leftarrow \cdots \leftarrow X_{k}\right)$ and letting $f_{k}^{\prime}: X_{k}^{\prime}$ $\rightarrow X_{k-1}^{\prime}$ be induced from collapsing the last mapping cylinder to its base. $\lim _{\leftarrow}\left(X_{i}^{\prime}, f_{i}^{\prime}\right)$ is a compactification of $\operatorname{Map}\left(\mathrm{pt} \leftarrow X_{1} \leftarrow X_{2} \leftarrow \ldots\right)$ obtained by adding on a copy of $\lim \left(X_{i}, f_{i}\right)$. We will call this space (the completed contractible mapping cylinder $) \overleftarrow{\mathrm{CMap}^{+}}\left(\left(X_{i}, f_{i}\right)\right)$. If the spaces $X_{i}$ are ANR's, then $\mathrm{CMap}^{+}\left(\left(X_{i}, f_{i}\right)\right)$ is an AR since it is $\varepsilon$-dominated by the open mapping telescope for each $\varepsilon>0$. Compare with [Ch-S, p. 181]. This is often a convenient AR containing the inverse limit.

Definition 5.3. If $f: X \rightarrow Y$ is an approaching map and $A \subset X$ is a closed set, we say that $f \mid A$ represents a map, $f^{*}: A \rightarrow Y$, on $A$ if $f \mid A \times[0, \infty): A \times[0, \infty) \rightarrow$ $W$ extends to a continuous function $f^{+}: A \times[0, \infty] \rightarrow W$ by defining $f^{+}(a, \infty)=$ $f^{*}(a)$ for all $a \in A$.

The following lemma is the main technical result of this section.

LemMA 5.4. Let $X$ be a compactum and let $A \subset X$ be a closed set. Let $Y$ $=\lim \left(Y_{i}, f_{i}\right)$ where each $Y_{i}$ is an $A N R$ and each $f_{i}$ is an approximate fibration for $X$. If $\underline{\alpha}: X \rightarrow Y$ is an approaching map (with respect to $Y \hookrightarrow \mathrm{C} \operatorname{Map}^{+}\left(Y_{i}, f_{i}\right)$ ) which represents a map on $A$, then $\underline{\alpha}$ is homotopic through approaching maps representing the same map on $A$ to an approaching map $\underline{\beta}: X \rightarrow Y$ which represents $a$ map on $X$.

Proof. Let $p$ : $\operatorname{CMap}^{+}\left(Y_{i}, f_{i}\right) \rightarrow[0, \infty]$ be the natural map and let $\varphi_{t}$ : $\mathrm{CMap}^{+}\left(Y_{i}, f_{i}\right) \rightarrow \operatorname{CMap}^{+}\left(Y_{i}, f_{i}\right)$ be the map which retracts $\operatorname{CMap}^{+}\left(Y_{i}, f_{i}\right)$ onto $p^{-1}([0, t])$ via the projection of $Y$ to the $Y_{i}$ 's and mapping cylinder collapses.

Since $\underline{\alpha}$ is an approaching map, $\alpha: X \times[0, \infty) \rightarrow \operatorname{CMap}^{+}\left(Y_{i}, f_{i}\right)$ has the property that for each $k$ there exists $N_{k}$ such that $\alpha\left(X \times\left[N_{k}, \infty\right)\right) \subset p^{-1}([k+1, \infty])$. We may clearly assume that $N_{k}<N_{k+1}$ for all $k$ and that $\lim _{k \rightarrow \infty} N_{k}=\infty$. Let $\rho$ : $[0, \infty) \rightarrow[0, \infty)$ be a homeomorphism into with $\rho(k)=N_{k}$ for all $k . \alpha: X \times[0, \infty)$ $\rightarrow \mathrm{CMap}^{+}\left(Y_{i}, f_{i}\right)$ is homotopic to $\alpha^{\prime}$ where $\alpha^{\prime}(x, t)=\alpha(x, \rho(t)) . \alpha^{\prime}$ has the property that $p \circ \alpha^{\prime}(x, t)>t$ for all $t$. $\alpha^{\prime}$, in turn, is homotopic to $\alpha^{\prime \prime}$ defined by $\alpha^{\prime \prime}(x, t)=$ $\varphi_{t} \circ \alpha^{\prime}(x, t)$. Both homotopies preserve the map represented by $\alpha$ on $A$. We may drop the primes and assume that $p \circ \alpha(x, t)=t$ for all $t$. 
The map $\alpha: X \times[0, \infty) \rightarrow \operatorname{Map}\left(* \leftarrow Y_{1} \leftarrow Y_{2} \leftarrow \ldots\right)$ is proper. If $\alpha^{*}: A \rightarrow Y$ is the map represented by $\underline{\alpha} \mid A$, then there is a proper homotopy from $\alpha \mid A \times[0, \infty)$ to the map

$$
\alpha^{* *}: A \times[0, \infty) \rightarrow \operatorname{Map}\left(* \leftarrow Y_{1} \leftarrow Y_{2} \leftarrow \ldots\right)
$$

given by $\alpha^{* *}(a, t)=\varphi_{t} \circ \alpha^{*}(a)$. The restriction of the homotopy to $A \times\{t\}$ is obtained from $\varphi_{t} \circ(\alpha \mid A \times[t, \infty])$ by reparameterizing from $[t, \infty]$ to $[0,1]$. We may use the proper homotopy extension theorem to alter $\alpha$ so that $\varphi_{t} \circ \alpha(a, t)=$ $\left(\alpha^{*}(a), t\right)$ for all $t$. We may repeat the initial types of homotopies to regain the condition $p \circ \alpha(x, t)=t$ for all $t$.

We now define a homotopy $\underline{A}$ of approaching maps by

$$
\begin{aligned}
A_{s}(x, t)=\varphi_{t} \circ \alpha\left(x, n-\frac{1}{2}\right)+\frac{2}{2-s}\left[t-\left(n-\frac{1}{2}\right)\right] \\
\quad \text { for } t \in\left[n-\frac{1}{2}, n+\frac{1-s}{2}\right], \\
=\varphi_{t} \circ \alpha\left(x, n+\frac{1}{2}\right) \quad \text { for } t \in\left[n+\frac{1-s}{2}, n+\frac{1}{2}\right] .
\end{aligned}
$$

Again, we write $\alpha(x, t)=A_{1}(x, t)$. $\alpha$ now has the property that $\alpha(x, t)=$ $\varphi_{t} \circ \alpha\left(x, n+\frac{1}{2}\right)$ for $t \in\left[n, n+\frac{1}{2}\right]$. We can think of $\alpha$ as being constructed from maps $\alpha_{n}: X \rightarrow Y_{n}$ (here identified with $\left.p^{-1}\left(n-\frac{1}{2}\right)\right)$ and homotopies $h_{n}: X \times I \rightarrow$ $Y_{n-1}$ from $f_{n} \circ \alpha_{n}$ to $\alpha_{n-1}$. The formulas are $\alpha_{n}(x)=\alpha\left(x, n-\frac{1}{2}\right)$ and $h_{n}(x, t)=$ $\varphi_{n-1 / 2} \circ \alpha(x, n-t / 2)$. By construction, $h_{n}(a, t)=\varphi_{n-1 / 2} \circ \alpha^{*}(a)$ for all $a \in A$ and for all $t \in[0,1]$.

Let $\left\{\varepsilon_{i}^{\prime}\right\}$ be a sequence of real numbers as in Lemma 4.1. Now choose $\varepsilon_{i}<\varepsilon_{i}^{\prime}$ so small that maps into $Y_{i}$ which are $\varepsilon_{i}$-close are canonically $\varepsilon_{i}^{\prime}$-homotopic. We start our construction by considering the diagram below.

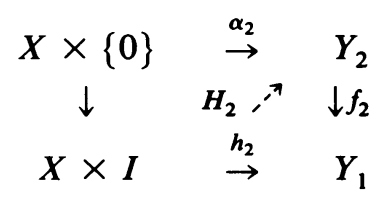

$h_{2}(x, 0)=f_{2} \circ \alpha_{2}$, so there is a homotopy $H_{2}: X \times I \rightarrow Y_{2}$ so that $H_{2}(x, 0)=$ $\alpha_{2}(x)$ and $d\left(f_{2} \circ H_{2}, h_{2}\right)<\varepsilon_{1}$. Let $H_{2}(x, 1)=\alpha_{2}^{\prime}(x)$. Since $h_{2} \mid(A \times I)$ is a constant homotopy, the usual regularizing trick for Hurewicz fibrations [D] allows us to assume that $H_{2}(a, t)=\alpha_{2}(a)$ for all $a \in A$ and $t \in[0,1]$. Set $\alpha_{1}^{\prime}=\alpha_{1}$ and let $h_{2}^{\prime}$ : $X \times I \rightarrow Y_{1}$ be a small canonical homotopy from $f_{2} \circ \alpha_{2}^{\prime}$ to $\alpha_{1}^{\prime}=\alpha$. Note that there is a canonical homotopy $G_{2}$ from $f_{2} \circ H_{2}$ to $h_{2}$ which extends $h_{2}^{\prime}$.

We have a homotopy $K_{2}$ from $f_{3} \circ \alpha_{3}$ to $\alpha_{2}^{\prime}$ given by

$$
K_{2}(x, t)= \begin{cases}h_{3}(x, 2 t), & 0<t \leqslant \frac{1}{2}, \\ H_{2}(x, 2 t-1), & \frac{1}{2} \leqslant t \leqslant 1 .\end{cases}
$$

Let $H_{3}: X \times I \rightarrow Y_{3}$ be an $\varepsilon_{2}$-lifting of $K_{2}$ starting at $\alpha_{3}$ and ending at $\alpha_{3}^{\prime}: X \rightarrow Y_{3}$. As before, let $h_{3}^{\prime}$ be a canonical homotopy from $f_{3} \circ \alpha_{3}^{\prime}$ to $\alpha_{2}^{\prime}$ and let $G_{3}$ be a 
canonical homotopy from $f_{3} \circ H_{3}$ to $K_{2}$. Continue in this fashion, letting $K_{n-1}=$ $h_{n} * H_{n-1}: X \times I \rightarrow Y_{n-1}$, letting $H_{n}$ be an $\varepsilon_{n-1}$-lifting of $K_{n-1}$, and letting $G_{n}$ be a canonical homotopy from $f_{n} \circ H_{n}$ to $K_{n-1}$ extending a canonical homotopy $h_{n}^{\prime}$ from $f_{n} \circ \alpha_{n}^{\prime}$ to $\alpha_{n-1}^{\prime}$, where $\alpha_{n}^{\prime}=H_{n}(x, 1)=K_{n}(x, 1)$.

Use the maps $\alpha_{n}^{\prime}$ and homotopies $h_{n}^{\prime}$ to define an approaching map $\alpha^{\prime}: X \times$ $[0, \infty) \rightarrow \mathrm{C} \mathrm{Map}^{+}\left(X_{i}, f_{i}\right)$ by the formula

$$
\alpha^{\prime}(x, t)= \begin{cases}\left(h_{n}^{\prime}\left(x, 1-2\left(t-n+\frac{1}{2}\right)\right), t\right), & t \in\left[n-\frac{1}{2}, n\right], \\ \left(\alpha_{n+1}^{\prime}(x), t\right), & t \in\left[n, n+\frac{1}{2}\right] .\end{cases}
$$

This simply reverses the process by which the original $\alpha_{n}$ 's and $h_{n}$ 's were defined. By Lemma 4.1, $\alpha^{\prime}$ represents a map which exends $\alpha^{*}$.

It remains to show that $\underline{\alpha}^{\prime}$ is homotopic to $\underline{\alpha}$. We have appropriate maps $H_{n}$ : $X \times I \rightarrow Y_{n}$. We need only produce homotopies $M_{n}$ from $f_{n} \circ H_{n}$ to $H_{n-1}$ extending $h_{n}$ and $h_{n}^{\prime}$ and use them to produce an approaching map as above. This amounts to extending a map from $X \times \partial I^{2}$ to $X \times I^{2}$, where the map on $\partial I^{2}$ is given schematically by the following diagram which shows only the $I^{2}$-coordinates.

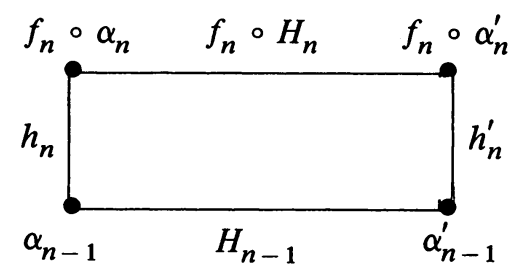

The extension is given by reparameterizing the homotopy $G_{n}$.

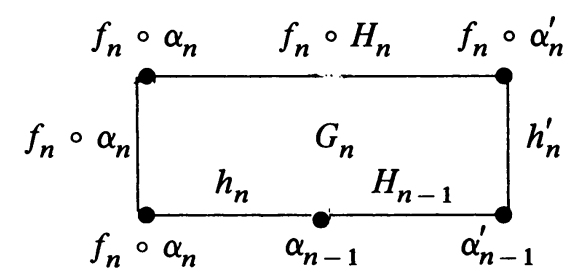

The unpleasant formula is left to the reader. This completes the proof of Lemma 5.4 .

Corollary 5.5. Let $Y=\lim _{\leftarrow}\left(Y_{i}, f_{i}\right)$ with each $Y_{i}$ a compact $A N R$.

(i) If each $f_{i}$ is an approximate fibration, then for any compactum $Z$, homotopy classes of maps $Z \rightarrow Y$ are in 1-1 correspondence with strong shape morphisms $Z \rightarrow Y$.

(ii) If each $f_{i}$ is an $A F^{i}$-map, then the same conclusion holds for compact finite-dimensional $Z$.

Proof. We prove part (ii). If $\operatorname{dim} Z=N$, then

$$
Y=\lim _{\leftarrow}\left(Y_{N} \leftarrow Y_{N+1} \leftarrow \ldots\right),
$$


so Lemma 5.4 applies to maps of $Z$ or $Z \times I$ into $Y$. Maps in a strong shape class are obtained by applying the lemma to $Z$. Homotopies are obtained by applying the lemma to $Z \times I$ relative to $Z \times\{0,1\}$.

REMARK. One can define simplicial sets of maps $Z \rightarrow Y$ and approaching maps $Z \rightarrow Y$. The proof above shows that the inclusion is a homotopy equivalence.

Theorem 3 now follows as in the discussion following Corollary 4.2. In fact, we can prove the slightly sharper result:

THEOREM 3'. If $Y=\lim \left(Y_{i}, f_{i}\right)$ with each $Y_{i}$ an $A N R$ and the homotopy fiber of each map $f_{i}$ has finite skeleta, then there is a compactum $Y^{\prime}$ shape equivalent to $Y$ which is improved in the sense of Theorem 3 and Corollary 5.5(ii).

Proof. Apply Theorem 2 to $f_{i} \times$ id: $Y_{i} \times Q \rightarrow Y_{i-1} \times Q$.

We can prove a result which does not follow from Theorem $3^{\prime}$ by appealing to Theorem 1 rather than to Theorem 2. A compactum $X$ is $L C^{k}$ if for each $x \in X$ and neighborhood $U$ of $x$ there is a neighborhood $V$ of $x$ so that every map $S^{l} \rightarrow V, 0 \leqslant l \leqslant k$, is nullhomotopic in $U$.

THeOrem 3". If $Y=\lim _{\leftarrow}\left(Y_{i}, f_{i}\right)$ with each $Y_{i}$ an $A N R$ and each $f_{i}(i+1)-$ connected, then $Y$ is shape equivalent to a compactum $Y^{\prime}$ which is improved in the sense of Theorem 3'. In addition, $Y^{\prime}$ is $L C^{k}$ for each $k>0$.

Proof. Apply Theorem 1 to construct a homotopy from $f_{i} \times$ id: $Y \times Q_{i} \rightarrow Y_{i-1}$ $\times Q$ to a $U V^{i}$-map $f_{i}^{\prime}$ and let $Y^{\prime}=\lim \left(Y_{i} \times Q, f_{i}^{\prime}\right)$. This proves Theorem $3^{\prime \prime}$ modulo the last assertion, which follows from the next proposition.

Proposition 5.6. Let $Y=\lim \left(Y_{i}, f_{i}\right)$ with each $Y_{i}$ an $A N R$. If all but finitely many $f_{i}^{\prime}$ 's are $U V^{k}$-maps, then $Y$ is $\overleftarrow{L} C^{k}$.

Proof. Let $y=\left(y_{1}, y_{2}, \ldots\right) \in Y$. Let $p_{i}: Y \rightarrow Y_{i}$ be the natural projection. The collection $\left\{p_{i}^{-1}(U) \mid U\right.$ is open in $\left.Y_{i}, i \geqslant K\right\}$ is a basis of $Y$ for each $K$, so given a neighborhood $U$ of $y$ we may find a neighborhood $U_{K}$ of $y_{K}$ in $Y_{K}$ so that $y \in p_{K}^{-1}\left(U_{K}\right) \subset U$. $K$ may be chosen so that $f_{i}$ is a $U V^{k}$-map for each $i>K$. Choose $V_{K} \subset U_{K}$ so that maps $S^{l} \rightarrow V$ are nullhomotopic in $U_{K}$ for $0<l \leqslant k$. $V_{K}$ exists because $Y_{K}$ is $L C^{k}$. Set $V=p_{K}^{-1}\left(V_{K}\right)$.

If $\alpha: S^{l} \rightarrow V, 0 \leqslant l \leqslant k$, let $\alpha_{n}=p_{n} \circ \alpha$ for each $n$. Since $\alpha_{K}: S^{l} \rightarrow V_{K}$, there is an extension $\beta_{K} \mid D^{l+1} \rightarrow U_{K}$ of $\alpha_{K}$. If a sequence $\left\{\varepsilon_{i}\right\}$ of positive reals is chosen as in Lemma 4.1, we can find a map $\beta_{K+1}: D^{l+1} \rightarrow Y_{K+1}$ extending $\alpha_{K+1}$ so that $d\left(f_{K+1} \circ \beta_{K+1}, \beta_{K}\right)<\varepsilon_{K}$. This lifting is possible because $f_{K}$ is $U V^{k}$. Continuing this process, we obtain a sequence $\left\{\beta_{i}: D^{l+1} \rightarrow Y_{i}\right\}_{i=K}^{\infty}$ of maps extending the maps $\alpha_{i}$ such that $d\left(f_{i} \circ \beta_{i}, \beta_{i-1}\right)<\varepsilon_{i-1}$ for $i \geqslant K+1$. As in Lemma 4.1, this defines a map $\beta$ into $Y$ extending $\alpha$.

REMARK. The condition that a space be $L C^{k}$ for all $k$ is not as nice as it seems at first glance. The one-point compactification of $\bigvee_{i=1}^{\infty} S^{i}$ has this property and is, in fact, an 'improved' compactum.

A compactum is strange if it has the shape of an infinite CW complex. Strange compacta were first constructed by Edwards and Geoghegan in [E-G]. (See [F $]$ for the analogous construction in homotopy theory.) 
COROllary 5.7. If $X$ is a strange compactum, then $X$ is shape equivalent to an improved compactum which is $L C^{k}$ for each $k>0$.

Proof. Let $K$ be a CW complex shape equivalent to $X . K$ is finitely dominated in the sense of Wall [E-G], so for each $i$ there exist a finite polyhedron $K_{i}$ and $(i+1)$-connected maps $d_{i}: K_{i} \rightarrow K, u_{i}: K \rightarrow K_{i}$ such that $d_{i} \circ u_{i} \simeq$ id. See [W] for a proof of this. The inverse system $\left\{\left(K_{i}, u_{i} \circ d_{i+1}\right)\right\}$ satisfies the hypothesis of Theorem $3^{\prime \prime}$ and has inverse limit shape equivalent to $X$ by [E-G]. Theorem 3" produces the desired compactum.

We remark that the compacta generated by Theorem 3' do not have good local properties. The dyadic solenoid is already improved in the sense of Theorem $3^{\prime}$.

Theorem 1 and Proposition 5.6 allow us to give a complete algebraic characterization of metric continua which are shape equivalent to $L C^{n}$ continua.

TheOrem 4. A continuum $X$ is shape equivalent to an $L C^{n}$ continuum if and only if pro- $\pi_{l}(X)$ is stable for $0 \leqslant l \leqslant n$ and Mittag-Leffler for $l=n+1$.

Proof. The argument of Kozlowski and Segal [K-S] shows that an $L C^{n}$ metric spaces have stable pro- $\pi_{l}$ for $l \leqslant n$. Borsuk [D-S, 5.2.8] has shown that $L C^{n}$ metric continua have Mittag-Leffler pro- $\pi_{n+1}$. For completeness and variety we sketch a proof which does not explicitly use refinements of nerves. Let $X=\lim \left(K_{i}, f_{i}\right)$ and form $\mathrm{CMap}^{+}\left(K_{i}, f_{i}\right)$. Triangulate the open telescope by finer and finer triangulations going out toward $X$. Use the $L C^{n}$ property to define a retraction of the $(n+1)$-skeleton to $X$ in some neighborhood of $X$. Since $\mathrm{CMap}^{+}\left(K_{i}, f_{i}\right)$ is an ANR, given any neighborhood $U$ of $X$ there is a neighborhood $V$ of $X$ so that $r \mid(V \cap$ $(n+1)$-skeleton) $\cup X$ is homotopic to the identity by a homotopy which fixes $X$. We claim that $\operatorname{im}\left(\pi_{l}(V) \rightarrow \pi_{l}(U)\right)$ is isomorphic to $\pi_{l}(X)$ for $l \leqslant n$ and that $\pi_{n+1}(X) \rightarrow \operatorname{im}\left(\pi_{n+1}(V) \rightarrow \pi_{n+1}(U)\right)$ is a surjection. If $\left(S^{l}, *\right) \stackrel{\alpha}{\rightarrow}(V, *)$, then by simplicial approximation we may assume that $\alpha\left(S^{l}\right) \subset(V \cap(n+1)$-skeleton $) \cup$ $X$ for $l \leqslant n+1$. $r \circ \alpha\left(S^{l}\right) \subset X$ and $r \circ \alpha \sim \alpha$ rel ${ }^{*}$ in $U$. This establishes the surjectivity of $\pi_{1}(X) \rightarrow \operatorname{im}\left(\pi_{1}(V) \rightarrow \pi_{1}(U)\right)$. If $\alpha:\left(S^{l}, *\right) \rightarrow(X, *)$ is nullhomotopic as a map $\alpha:\left(S^{l}, *\right) \rightarrow(U, *), l \leqslant n$, let $\beta:\left(D^{l+1}, *\right) \rightarrow(U, *)$ be an extension of $\alpha$. We assume as before that $\beta\left(D^{l+1}\right) \subset((n+1)$-skeleton $) \cap U$, so $r \circ \beta: D^{l+1} \rightarrow X$, * is an extension of $\alpha$. This proves injectivity.

Thus, we need only prove the reverse implication. The idea is to show that a continuum $X$ satisfying the stated conditions can be represented as an inverse limit of ANR's with $(n+1)$-connected bonding maps. ${ }^{4}$ We will then cross with $Q$ and apply Theorem 1 to construct an inverse sequence with $U V^{n}$ bonding maps and inverse limit shape equivalent to $X$. We proceed by induction on $n$.

Case $n=0$. We can represent $X$ as an inverse $\operatorname{limit} \lim \left(K_{i}, f_{i}\right)$ of connected polyhedra where $f_{i^{*}}$ maps $f_{i+1^{*}}\left(\pi_{1}\left(K_{i+1}\right)\right)$ onto $f_{i^{*}}\left(\pi_{1}\left(K_{i}\right)\right)$ for each $i$, the last part being essentially the Mittag-Leffler condition. For each $i$, let $\left\{{ }^{i} g_{j}\right\}_{j=1}^{N_{i}}$ be a finite generating set for $\pi_{1}\left(K_{i}\right)$ and for each $j$ choose $i h_{j} \in f_{i+1 *}\left(\pi_{1}\left(K_{i+1}\right)\right)$ such that

\footnotetext{
${ }^{4}$ Compare with [H-I].
} 
$f_{i^{*}}\left({ }^{i} g_{j}\right)=f_{i_{*}}\left({ }^{i} h_{j}\right)$. Attach 2-cells to $K_{i}$ to kill the elements ${ }^{i} g_{j}\left({ }^{i} h_{j}\right)^{-1}$ and call the resulting complex $K_{i}^{\prime}$.

Since $f_{i^{*}}\left({ }^{i} g_{j}^{i} h_{j}^{-1}\right)=1$ for each $j, f_{i}$ extends to a map $k_{i}^{\prime}: K_{i}^{\prime} \rightarrow K_{i-1}$. Setting $f_{i}^{\prime}$ equal to the composition of $k_{i}^{\prime}$ with $K_{i-1} \rightarrow K_{i-1}^{\prime}$, we have a strictly commuting diagram of polyhedra.

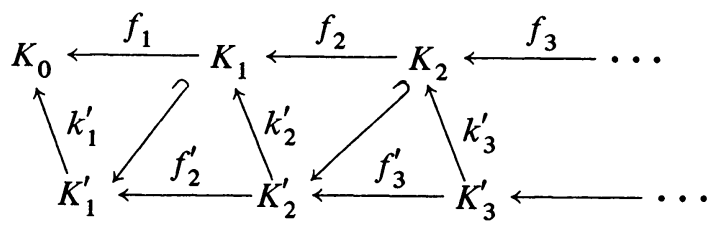

One can check that each $f_{i^{*}}^{\prime}: \pi_{1}\left(K_{i}^{\prime}\right) \rightarrow \pi_{1}\left(K_{i-1}^{\prime}\right)$ is surjective. A word represented by a string of ${ }^{i} g_{j}$ 's is the same as the word represented by the same string of ${ }^{i} h_{j}$ 's in $\pi_{1}\left(K_{i}^{\prime}\right)$. Since the diagram strictly commutes, $\underset{\leftarrow}{\lim }\left(K_{i}^{\prime}, f_{i}^{\prime}\right)=X$. This completes the case $n=0$. Note that if pro- $\pi_{1}$ is stable (i.e. if im $f_{i^{*}}$ maps isomorphically to $\operatorname{im} f_{i^{*}} \circ f_{i+1^{*}}$ for large $i$ ), as in the succeeding cases, then all but finitely many of the maps $f_{i}^{\prime}$ induce isomorphisms on $\pi_{1}$.

Case $n=1$. In this case we have (dropping primes) isomorphisms $\pi_{1}\left(K_{i}\right) \stackrel{\approx}{\rightarrow} \pi_{1}\left(K_{i-1}\right)$ for all $i$ and a commuting diagram.

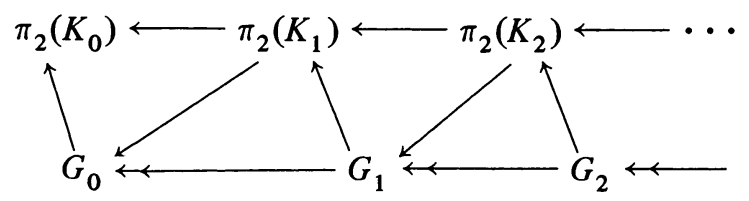

We have an exact sequence $\pi_{2}\left(K_{i}\right) \rightarrow \pi_{2}\left(K_{i-1}\right) \rightarrow \pi_{2}\left(M\left(f_{i}\right), K_{i}\right) \rightarrow 0$. Since we have $\pi_{1}$-isomorphisms, we can pass to universal covers and get:

$$
\pi_{2}\left(M\left(f_{i}\right), K_{i}\right)=\pi_{2}\left(\widetilde{M\left(f_{i}\right)}, \tilde{K}_{i}\right)=H_{2}\left(M\left(\tilde{f}_{i}\right), \tilde{K}_{i}\right) .
$$

$C_{*}\left(M\left(\tilde{f}_{i}\right), \tilde{K}_{i}\right)$ is a complex of finitely generated free $Z_{\pi_{1}}(K)$-modules. The first nonvanishing homology group of such a complex is always finitely generated. See [W], [Si], or the proof of [Co, 13.1] for a proof of this. Choose a finite set of generators $\left\{\bar{\alpha}_{j}\right\}$ for $\pi_{2}\left(M\left(f_{i}\right), K_{i}\right)$ and for each $j$ let $\left\{\alpha_{j}^{\prime}\right\}$ be a representative for $\bar{\alpha}_{j}$ in $\pi_{2}\left(K_{i-1}\right)$. Using the Mittag-Leffler condition, we see that for each $j$ there is a $\beta_{j} \in \pi_{2}\left(K_{i}\right)$ such that $f_{i-1^{*}} \circ f_{i^{*}}\left(\beta_{j}\right)=f_{i-1^{*}}\left(\alpha_{j}^{\prime}\right)$. Let $\alpha_{j}=\alpha_{j}^{\prime}-f_{i^{*}} \beta_{j} . \alpha_{j}$ maps to $\bar{\alpha}_{j}$ in the cokernel and $f_{i-1^{*}}\left(\alpha_{j}\right)=0$ for each $j$. We can now attach finitely many 3-cells to $K_{i}$ to kill $\left\{\alpha_{j}\right\}$ and create a system $\left\{\left(K_{i}^{\prime}, f_{i}^{\prime}\right)\right\}$ with inverse limit shape equivalent to $X$ and 2-connected bonding maps.

Case $n \geqslant 2$. We assume that we have produced a system with $n$-connected bonding maps. The system $\left\{\left(\pi_{n}\left(K_{i}\right), f_{i^{*}}\right)\right\}$ is a system of surjections and is stable, so an easy argument shows that it is a sequence of isomorphisms beyond some point. We now proceed to the $(n+1)$ st homotopy and kill the cokernels as in the case $n=1$. 
The case $n=0$ of Theorem 4 is a result of Krasinkiewicz [K]. Our proof of Theorem 1 is somewhat crude, so the $L C^{n}$ compacta we produce are infinite-dimensional. In case $n=0$, a theorem of John Walsh [ $\mathbf{W a}_{1}$ ] can be substituted to give finite-dimensional $L C^{0}$ continua when $X$ is finite dimensional.

For $n=\infty$ we see that $Y$ has the shape of a continuum which is $L C^{k}$ for all $k \Leftrightarrow$ pro- $\pi_{l}(Y)$ is stable for all $l \Leftrightarrow$ the conditions of Theorem 3 " are satisfied. Are these spaces shape equivalent to locally contractible spaces?

\section{An application to $\mathrm{CE}$ equivalence.}

Definition 6.1. If $X$ and $Y$ are compacta, we say that $X$ is CE equivalent to $Y$ if there exist compacta $X=X_{0}, X_{1}, \ldots, X_{n}=Y$ and for each $i$ either $X_{i+1} \stackrel{\text { CE }}{\rightarrow} X_{i}$ or $X_{i} \stackrel{\mathrm{CE}}{\rightarrow} X_{i+1}$. (The sophisticate will want to use hereditary shape equivalences [D-S] in this definition. We will work with the simpler notion and point out that the problems discussed here are still interesting when all of the spaces involved are required to be finite dimensional, in which case CE maps are hereditary shape equivalences.)

In $\left[\mathbf{F}_{\mathbf{1}}\right]$, the author showed that homotopy equivalent compacta are $\mathrm{CE}$ equivalent. Our attempt to prove that shape equivalent compacta are CE equivalent was blocked by a counterexample; in $\left[F_{2}\right]$ we showed that the spiral $X$ is not CE equivalent to $S^{1}$.

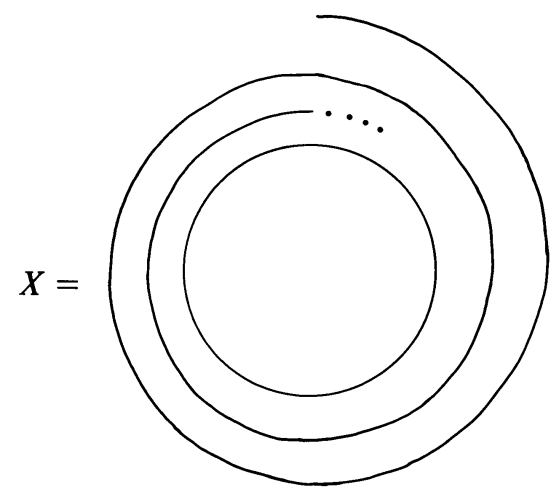

Note that $X$ is the 'mapping cylinder' of an approaching map $* \rightarrow S^{1}$. Our interest in approaching maps was stimulated by an attempt to develop a calculus of mapping cylinders in the shape category and imitate the proof in $\left[\mathbf{F}_{\mathbf{1}}\right]$.

Definition 6.2. If $f: X \rightarrow Y$ is an approaching map, $Y \subset W^{\mathrm{AR}}$, such that $f(X \times[0, \infty)) \subset W-Y$ and $f: X \times[0, \infty) \rightarrow W-Y$ is an imbedding, then the set $f(X \times[0, \infty)) \cup Y$ is called the mapping cylinder of $f$ and is denoted by $M(f)$. If $Y$ is imbedded as a $Z$-set [Ch] in $Q$, then every approaching map $f: X \rightarrow Y$ can be perturbed slightly to one which admits a mapping cylinder. It is easy to extend this definition so that all approaching maps have well-defined mapping cylinders by using such perturbations, since mapping cylinders obtained by different perturbations are homeomorphic. 
Proposition 6.3. If $K$ and $L$ are finite polyhedra with $\pi_{1} L=0$ and $f: K \rightarrow L$ is an approaching map which is homotopic to a constant map, then $M(f)$ is ${ }^{-} C E$ equivalent to $L$.

REMARK. No pair of shape equivalent CE-inequivalent $U V^{1}$ compacta is known. Proposition 6.3 shows that one cannot construct such an example by, for example, letting a ray spiral toward a copy of $S^{2}$. Note that the spiral $X$ shows that the condition $\pi_{1} L=0$ is necessary.

Proof (Proposition 6.3). Let $\operatorname{dim} K=k$. By Theorem 2 ' there exist a contractible polyhedron $P$ and a PL $A F^{k}$-map $P \rightarrow L$. By taking a regular neighborhood of $P$ in $R^{N}, N$ large, we may assume that $P$ is a disc. Let $r: U \rightarrow L$ be a retraction of a neighborhood of $L$ onto $L$. We may extend $r$ to be defined on $f(X \times[0, \infty))$. We have a diagram.

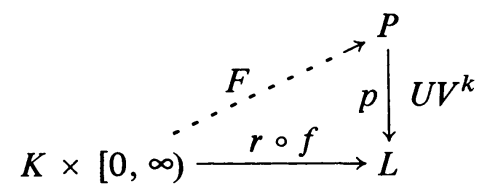

Since $r \circ f \mid K \times\{0\}$ is nullhomotopic, there is a lifting $F: K \times[0, \infty) \rightarrow P$ such that $\lim _{t \rightarrow \infty} d(p \circ F(x, t), r \circ f(x, t))=0$.

Imbed $P$ as a $Z$-set in $Q$ and perturb the approaching map $\underline{F}: K \rightarrow P$ so that it admits a mapping cylinder. Now consider the space $M(p) \cup_{P} M(\underline{F}) . M(\underline{F})$ is cell-like, so there is a CE-map $M(p) \cup_{P} M(\underline{F}) \rightarrow M(p) / P \cong M(p)$, since $P$ is a disc. Since $M(p) \stackrel{\text { CE }}{\rightarrow} L, M(p) \cup_{P} M(\underline{F})$ is CE equivalent to $L$. On the other hand, one can collapse the rays of $M(p)$ without first contracting $M(\underline{F})$. The resulting quotient space is homeomorphic to $M(f)$. This completes the proof of Proposition 6.3.

If $L$ is an $(r+1)$-connected polyhedron then a map $p t \rightarrow L$ is $(r+1)$-connected and, by Theorem $2^{\prime}, L$ is the $U V^{r}$-image of a contractible polyhedron. If $Y$ is a compactum $\mathrm{CE}$ equivalent to $L$ (using hereditary shape equivalences) then an argument using pullbacks $\left[\mathbf{F}_{2}\right]$ shows that $Y$ must be the $U V^{r}$-image of a cell-like set. This property was used in $\left[F_{2}\right]$ to show that the spiral $X$ is not $C E$ equivalent to $S^{1}$. The next proposition shows that this invariant cannot distinguish a mapping cylinder from its base when the base is simply connected.

Proposition 6.4. If $f: K \rightarrow L$ is an approaching map, and $L$ is $(s+1)$-connected, $s \geqslant 0$, then $M(f)$ is the $U V^{s}$-image of a cell-like set.

Proof. Let $r: U \rightarrow L$ be a retraction as before. Since $L$ is $(s+1)$-connected, the restriction $r \circ f \mid K^{(s+1)} \times\{0\}$ is nullhomotopic. The inclusion $K^{(s+1)} \rightarrow K$ is $(s+1)$-connected, so there exist a polyhedron $\bar{K}$ and a $U V^{s}$-map $\bar{i}: \bar{K} \rightarrow \bar{K}$ so that $r \circ f \circ \bar{i}$ is nullhomotopic. Letting $P$ be as before, we have a diagram. 


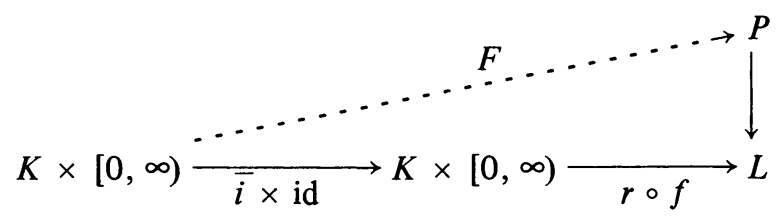

As before, we lift to $F$ and form $M(\underline{F})$. There is an obvious $U V^{s}$-map from the cell-like set $M(\underline{F})$ onto $M(f)$.

The place where these arguments break down when $L=S^{1}$ is that there is no $A F^{n}$-map from a contractible polyhedron to $L$ for $n \geqslant 0$. That is because the homotopy fiber of $p t \rightarrow S^{1}$ is an infinite discrete set. This is exploited by the covering space argument in $\left[\mathbf{F}_{2}\right]$. This suggests (to the author, at least) that $U V^{1}$, shape equivalent, $\mathrm{CE}$ inequivalent compacta may well exist but that to detect them one will need to exploit the fact that the homotopy fiber of $p t \rightarrow L$ frequently has infinitely generated homology when $\pi_{1} L=0$. This will require much more delicate arguments than those of $\left[\mathbf{F}_{\mathbf{2}}\right]$.

\section{REFERENCES}

[Bo] K. Borsuk, Theory of shape, Monografie Mat., vol. 59, Polish Science Publ., Warszawa, 1975.

[B] M. Brown, Some applications of an approximation theorem for inverse limits, Proc. Amer. Math. Soc. 11 (1960), 478-483.

[Ch] T. A. Chapman, Notes on Hilbert cube manifolds, CBMS Regional Conf. Ser. in Math., no. 28, Amer. Math. Soc., Providence, R. I., 1976.

[Ch-S] T. A. Chapman and L. Siebenmann, Finding a boundary for a Hilbert cube manifold, Acta Math. 137 (1976), 171-208.

[Co] M. M. Cohen, A course in simple homotopy theory, Springer-Verlag, New York, 1970.

[C-D] D. Coram and P. Duvall, Approximate fibrations and a movability condition for maps, Pacific J. Math. 72 (1977), 41-56.

[D-T] A. Dold and R. Thom, Quasifaserungen und unendliche symmetrische Producte, Ann. of Math. (2) 67 (1958), 239-281.

[D] J. Dugundji, Topology, Allyn and Bacon, Boston, Mass., 1966.

[D-S] J. Dydak and J. Segal, Strong shape theory (preprint).

[E-G] D. Edwards and R. Geoghegan, Shapes of complexes, ends of manifolds, homotopy limits and the Wall obstruction, Ann. of Math. (2) 101 (1975), 521-535.

[E-H] D. Edwards and H. Hastings, Cech and Steenrod homotopy theories with applications to geometric topology, Lecture Notes in Math., vol. 542, Springer-Verlag, Berlin and New York, 1976.

[F 1 S. Ferry, Homotopy, simple homotopy, and compacta, Topology (to appear).

$\left[\mathrm{F}_{2}\right] \ldots$, Shape equivalence does not imply $C E$ equivalence, Proc. Amer. Math. Soc. (to appear).

[K-O] Y. Kodama and Y. Ono, On fine shape theory. I, II, Fund. Math. (to appear).

[K-S] G. Kozlowski and J. Segal, Local behavior and the Vietoris and Whitehead theorems in shape theory, Fund. Math. 99 (1978), 213-225.

[K] J. Krasinkiewicz, Local connectedness and pointed 1-movability, Bull. Acad. Polon. Sci. Sér. Sci. Math. Astronom. Phys. 25 (1977).

[L] R. Lacher, Cell-like mappings and their generalizations, Bull. Amer. Math. Soc. 83 (1977), 495-552.

[M-R] S. Mardesic and T. B. Rushing, $n$-shape fibrations (preprint).

[Q] J. Quigley, An exact sequence from the nth to the $(n-1)$ st fundamental group, Fund. Math. 77 (1973), 195-210.

[Si] L. C. Siebenmann, The obstruction to finding a boundary for an open manifold of dimension $m>5$, Thesis, Princeton University, 1965.

[Sm] S. Smale, A Vietoris mapping theorem for homotopy, Proc. Amer. Math. Soc. 8 (1957), 604-610.

[S] E. Spanier, Algebraic topology, McGraw-Hill, New York, 1966.

[W] C. T. C. Wall, Finiteness conditions for CW complexes, Ann. of Math. (2) 81 (1965), 55-69.

[Wa $\mathbf{a}_{1}$ J. Walsh, Monotone and open mappings on manifolds. I, Trans. Amer. Math. Soc. (to appear). 
[Wa ] _ Light open and open mappings on manifolds. II (preprint).

[Wh] J. H. C. Whitehead, Simplicial spaces, nucleii, and m-groups, Proc. London Math. Soc. 45 (1939), 243-327.

[Wi $]$ D. Wilson, Open mappings on manifolds and a counterexample to the Whyburn conjecture, Duke Math. J. 40 (1973), 705-716.

$\left[\mathrm{Wi}_{2}\right]$ (1974), 103-109.

[H-I] L. Husch and I. Ivansic, Shape domination and imbedding up to shape (preprint).

School of Mathematics, Institute for Advanced Study, Princeton, New Jersey 08540

Department of Mathematics, University of Kentucky, LeXington, Kentucky 40506 (Current address) 